\title{
Internet-Based Health Promotion and Disease Control in the 8 Cities: Successes, Barriers, and Future Plans
}

\author{
MARY MCFARLANE, PHD, ${ }^{*}$ RACHEL KACHUR, MPH, CHES, ${ }^{*}$ JEFFREY D. KLAUSNER, MD, MPH, $\dagger$ \\ ERIC ROLAND, $\ddagger$ AND MARC COHEN§
}

\begin{abstract}
Objectives: The objective of this paper is to provide a detailed description of Internet-based sexually transmitted disease/human immunodeficiency virus prevention in the 8 US cities most affected by syphilis in men who have sex with men.

Goal: By reviewing the efforts under way in these 8 cities, we will understand the barriers and facilitators associated with Internet-based prevention efforts.

Study: This is a review of Internet activities taking place in $\mathbf{8}$ major US cities.

Results: Efforts in the 8 cities vary, with some cities reporting little or no Internet-based prevention activities. Other cities have attempted banner advertising, online outreach, online partner notification, online laboratory slips for syphilis testing, and auditorium-style chat sessions.

Conclusion: Though a number of policy-related barriers prevent some cities from engaging in Internet-based prevention, these activities are clearly important to the overall prevention effort. In order to surmount local policy barriers, it is essential to obtain evaluation data from the programs initiated.
\end{abstract}

BULL AND MCFARLANE" FOUND that the Internet "has a greater, more instantaneous reach than any other medium to facilitate encounters that result in sexual activity." Using the Internet to meet sex partners has been shown to be associated with an increased number of partners, including an increased number of partners known to be human immunodeficiency virus (HIV)-positive and increases in reported anal sex when compared with sex with partners met via other venues. ${ }^{2}$ In some cities in the United States and Europe, increases in the incidence of syphilis among men who have sex with men (MSM) are associated with increases in the use of the Internet to meet new sex partners. ${ }^{3,4}$ In addition, outbreaks of syphilis have been traced to Internet-based "chat rooms" in a number of cities, ${ }^{5,6}$ with a large proportion of contacts who are anonymous or known only by their chat-room nicknames. As the Internet becomes ever faster, cheaper, and more accessible, it is likely that Internet-initiated sexual activity will continue to be a factor in outbreaks of sexually transmitted diseases.

The public-health response to the issues raised by the Internet has been varied. In outbreak situations, the primary concerns of health officials are partner notification, testing of contacts, and treatment of cases. Partner notification on the Internet is made complicated by a baffling array of online security and anonymity protections, though some of the cities included in this report have made progress in these efforts. ${ }^{5}$ Testing can be facilitated online,

Correspondence: Mary McFarlane, PhD, 1600 Clifton Road NE, Mailstop E44, Atlanta, GA 30333. E-mail: xzm3@cdc.gov.

Received for publication December 27, 2004, and accepted May 9, 2005.
From the *Division of STD Prevention, National Center for HIV, STD, and TB Prevention, Centers for Disease Control and Prevention, Atlanta, Georgia; TSTD Prevention and Control Services, San Francisco Department of Health, San Francisco, California; ¥Montrose Clinic, Houston, Texas; and the §United Foundation for AIDS, Miami, Florida

but only 1 site has attempted online laboratory-slip interventions. ${ }^{7}$ At present, no national site details the locations in which individuals can receive free or low-cost syphilis tests. Cases and contacts can be referred for treatment via emails or outreach in chat rooms, but the efficacy of these interventions is largely unknown. ${ }^{8} \mathrm{Al}-$ though several health departments are in the early stages of implementing outbreak-control measures online, there is a general lack of evaluation and publication on the topic, with the exception of San Francisco. ${ }^{7}$

Another avenue of public-health response to the Internet involves disease prevention and health promotion. Health information has long been available online, but the quality of this information and the appropriateness of the online delivery can be questioned. Some gay-oriented websites, such as gay.com and gayhealth.com, have health channels embedded in their offerings. Still, these health-information pages represent passive, nonintrusive attempts at promoting health online. They require users to take the initiative to search for topics such as syphilis and other sexually transmitted diseases. Banner ads pointing users to these sites have been implemented ${ }^{7}$ and are being tried in other locations as well.

Despite the wide availability of health information online, few sites actively intervene upon MSM risk behaviors such as multiple partners or "barebacking" (i.e., anal sex without the use of a condom), or on pro-health behaviors such as sexually transmitted disease (STD) and HIV testing. Some of the areas most affected by syphilis in MSM accomplish health promotion by chat-room outreach, described herein. Tailored, personalized interventions, accomplished through brief surveys and algorithmic, programmable, automated responses are another option for online intervention. Though one such online intervention for MSM has been implemented and evaluated, ${ }^{9}$ the findings raise as many methodological questions about Internet interventions as they answer.

Clearly, the sexual risk environment of the Internet presents a challenge for health officials involved in syphilis-elimination efforts. This report focuses on the Internet-based interventions that have been attempted in the 8 cities most affected by increases in syphilis incidence in MSM. 


\section{Methods}

Public health officials in each of the 8 cities (Chicago, New York, Miami, Fort Lauderdale, San Francisco, Los Angeles, Houston, and Atlanta) were contacted for interviews regarding local, online efforts to implement disease-control and health-promotion strategies. The initial contacts were selected by asking CDC program consultants to identify the senior public health official most likely to know about Internet-related efforts in the local area. Often, the initial interviews resulted in a snowball sample of local experts in this field. In some cases, such as Chicago, Houston, Miami, and San Francisco, health department staff referred the interviewer to local community partners who performed the health department's Internet-related work.

These qualitative, open-ended interviews were conducted using a topic guide that covered online partner notification, testing slips, banner advertisements, chat-room outreach, automated behavioral interventions, and local, Internet-related epidemiology. In one instance, when the lead author was unable to interview a participant directly, the telephone interview with another author (RK) was recorded, transcribed without identifiers, and provided to the lead author. Where available, data were contributed by representatives of both public health programs and community-based organizations in each site involved. Though these data-collection efforts were not subject to review by institutional review boards due to their status as program evaluation activities, every effort was made to keep telephone calls confidential and to keep data secure.

\section{Results}

Online efforts can be divided into several categories. In general, local efforts begin in the context of an Internet-implicated outbreak. Disease intervention specialists (DIS), tasked with finding partners of persons diagnosed with syphilis, often encounter obstacles to partner notification online. In some instances, chat rooms are used to raise outbreak awareness and augment partner notification strategies. To further facilitate testing, one city has implemented online "test slips," i.e., a signed, online order for a laboratory to perform a syphilis test that can be redeemed in the local jurisdiction. In conjunction with these techniques, some of the cities have negotiated for banner advertisements in various chat rooms and Internet venues. Banner advertisements generally direct the user to a website that contains standard, didactic health communications, speaking to the need for interactive, targeted interventions. Because these have been the steps followed by several health departments with varying degrees of success, we will address each step in turn: online partner notification, chat-room outreach, online test slips, banner advertisements, and interactive, targeted interventions.

\section{Partner Notification on the Internet}

Most of the 8 sites have been forced to confront the idea of adapting traditional, face-to-face, partner-notification strategies to the Internet. In Chicago, as in many cities, health department staff are restricted from performing online partner notification by lack of computer access in clinics, lack of access to the Internet in most offices, firewalls protecting any Internet-enabled computers from gaining access to "sexual" websites, and local policy restricting use of the Internet by city employees for activities that can be construed as sexual. Fortunately, the Howard Brown Health Center, a community health facility that reports the largest number of syphilis cases in Chicago, is not so restricted and has worked with the health department to obtain its own DIS. Staff at Howard Brown routinely ask patients whether they have met sex partners online and use electronic mail (e-mail) and chat rooms to perform partner notification when warranted.

The use of e-mail is in many ways preferable to the use of live chat because of the differences in time required for finding partners. E-mail can be accomplished passively (i.e., one e-mail can be sent and the DIS can move on to other tasks while awaiting a response); however, locating someone in a chat room requires "lurking" for long periods in the chat rooms, hoping that the relevant person will log on. Furthermore, if only chat-room handles are known, it may be difficult to identify people at all, as many online sex seekers use multiple handles. Once contact is made with a partner, following advice from Howard Brown legal counsel, staff do not reveal to contacts that they have been exposed to syphilis, or even that they have been exposed to a disease at all. Rather, online contacts are told that staff have "important health information for you" and are encouraged to call or visit Howard Brown. Syphilis is mentioned only on the telephone or face to face, after the identity of the contact has been verified.

Though evaluation data have been gathered, the data collection has been sporadic, and no formal evaluation of Howard Brown's online partner-notification system exists. Of an initial 10 "online contacts" named during syphilis case interviews, 8 were found in chat rooms, and 7 of those presented to the DIS for diagnosis and treatment. Two tested positive for syphilis.

Other sites, such as Los Angeles, ${ }^{10}$ routinely request e-contact information during partner elicitation interviews. One person provided over 200 names of online contacts during one such interview. Because Los Angeles currently is in the process of evaluating online partner notification, those data are not yet available. However, initial observations indicate that the success of online partner notification is greatly enhanced when the original patient sends personal e-mail to the contacts. Although no Los Angeles-area contacts have had adverse reactions to online partner notification, some concern was expressed by local governments that HIPAA violations potentially could result from these efforts. Houston and San Francisco have conducted online outbreak-notification and partner-notification efforts. Houston reports mixed reactions from online contacts, and no actual evaluation data. A full evaluation of San Francisco's online efforts is presented elsewhere. ${ }^{5,7}$ In the course of their Internet-based partner notification efforts, San Francisco has developed a series of tips for conducting online partner notification; these are included in the box, reprinted from the MMWR.

Finally, Atlanta, New York, Miami, and Fort Lauderdale report no formal, online partner-notification efforts. In most sites, this is a result of a series of bureaucratic hurdles, including clearance of online protocols, concerns regarding privacy and confidentiality, and Internet access. Where Internet access is available, staff usually are prevented from using the Internet for partner-notification purposes by local policies and firewalls. If DIS need to conduct online investigations, they occasionally resort to using their home computers.

\section{Chat-Room Outreach}

Chat-room outreach currently occurs in San Francisco, Houston, Chicago, and the greater Miami/Fort Lauderdale area. Outreach in chat rooms involves individual staff members logging into chat rooms, often with a handle (or nickname) such as "letstalkaboutsex" or "askmeabouthealth." Staff members create "profiles" that explain the purpose of their visit to the chat rooms, the types of questions they can answer, and referral information for testing and treatment. Usually, the outreach staff are fairly passive in the chat rooms, with the exception of sending welcome messages to new arrivals and occasionally posting a brief line such as, "IM [instant 
message] me for sexual health info." This passivity prevents the chatters from becoming annoyed with the outreach staff, as has occurred in more active efforts.

Chicago's Howard Brown Health Center conducts chat-room outreach in venues such as manhunt.net, gay.com, and America Online (AOL). Outreach staff announce their presence and state that they are there to answer questions. The outreach staff target syphilis in particular but are trained to answer questions regarding any sexually transmitted disease. Though no evaluation has been conducted, an evaluation plan includes counting the number of contacts made during various times of day, descriptions of encounters, and number of referrals made. Because Chicago chat-rooms often include participants from other parts of Illinois, as well as Wisconsin and Indiana, it may not be possible to evaluate whether referrals result in clinic attendance.

In Houston, Montrose Clinic staff conduct online outreach as part of Project CORE (Cyber OutReach Education). A handbook has been developed for staff performing online interventions. The handbook contains material contributed by other sites and is a valuable resource for the project staff. The chat-room outreach in Houston is slightly more active than in Chicago in the sense that staff will occasionally post a topic, question, or statistic in an effort to generate contact with chatters. Staff use instant messaging, private chat, and larger "group" chat to accomplish their outreach. Referrals are made to other online resources, such as gayhealth.com, and to the Project CORE website with its full list of referrals. The website address is listed in the staff member's signature and profile.

One method of evaluating the Project CORE outreach includes counting the number of hits on Project CORE's website. A more intricate evaluation, involving qualitative analysis of all online conversations (saved electronically and without identifiers), is planned. As a major focus of the outreach is referral for STD testing, it is hoped that the referral system can be evaluated.

In San Francisco during 2 months of 2002, staff from the Department of Public Health and from Internet Sexuality Information Services, Inc. (ISIS) conducted chat-room outreach in 3 venues. These venues were AOL chat-rooms specific to San Francisco, Craigslist (San Francisco), and M4M4Sex. Topics addressed with chatters included symptoms, transmission and treatment of syphilis (as well as other STD, including HIV), and referral to testing. Staff responded to questions and provided syphilis fact sheets and online coupons for syphilis testing at the public STD clinic. In this location, a protocol guide and training manual were developed and an evaluation was conducted.

During the 2-month period, San Francisco outreach staff spent 57 hours conducting chat-room outreach in the 3 venues. They logged 212 interactions (67 on M4M4Sex, 21 on Craigslist, 124 on AOL). The rate of coupon redemption was $16 \%$ (35 coupons redeemed).

In another form of online outreach, San Francisco Department of Public Health (SFDPH) collaborated with ISIS to establish 7 auditorium-style chats with online visitors to gay.com. These chats were real-time, 1-hour interactions facilitated by a physician from SFDPH. Online chatters entered questions that were then selected by a moderator for expert response. The expert then posted an answer to the selected question. The software for conducting auditorium chat records the number of participants at any given time, and transcripts were reviewed and edited for clarity and removal of personal identifiers. Edited transcripts were posted on gay.com. During the 7, 1-hour auditorium chats, approximately 120 visitors per session attended, with 10 to 50 people in the room at any given time. Questions were answered at the rate of
15 per hour. It is not clear whether participants were from the San Francisco area or from elsewhere.

Though online outreach does not occur through the health department in Florida, a community-based organization (CBO) called United Foundation for AIDS (UFA) actively conducts Internet-based outreach to MSM. One focus of this outreach includes users of crystal methamphetamine ("crystal meth"). UFA developed the Crystal Alert program, focused on Internet users who use crystal meth, to get users to meet face to face to address risk issues. The program was developed in collaboration with an advisory committee of former users. The daily meetings have grown from approximately 7 to 10 members to 25 to 30 attendees. Other outreach focuses on bringing MSM to the health department for STD and HIV testing. The Miami outreach is possibly the most intensely personal type of outreach, occasionally going so far as to include sending staff members to drive people to the clinic for testing. When appropriate, outreach staff follow up with chatters who have been tested and provide a type of support throughout the process. Though the program has not been formally evaluated, transcripts of chats (without identifiers) have been kept for review. During the chats, staff have noted a desire on the part of chatters to understand what sexual behaviors are less risky than others, e.g., "Is a top less risky than a bottom? Is oral sex likely to get me infected?" These and other questions about the risks associated with particular sexual behaviors speak to the need for well-trained, expert outreach workers.

\section{Online Testing}

The SFDPH launched its online syphilis testing program in June 2003. Persons can log on to stdtest.org to obtain a physicianordered laboratory requisition ("lab slip") and a unique identification number. The lab slip, once printed out, can be taken to a number of local, private laboratories for specimen collection and analysis. When testing is complete, the results are provided to SFDPH, who takes responsibility for posting the results with the identification number on the website. The site also provides syphilis-related educational information, including signs, symptoms, and recommendations for future screening. Through mid-January of 2004, thousands of visits were logged on stdtest.org, but only 140 completed syphilis testing. Of the participants who completed testing, $6(4.3 \%)$ had new syphilis infections (4 infectious; 2 latent). Five of these infected patients were gay men. Of the gay men, 1 was HIV positive, 2 were HIV negative, and 2 were of unknown HIV serostatus. All infected patients received medical evaluation and treatment. ${ }^{7}$

\section{Banner Advertisements}

Considering that the Internet is a multimedia environment with vast reach to the US population, it is natural to consider broadbased, public-health communications online. Banner advertisements represent one such broad-based communication option. Banner advertisements are analogous to billboards in that they are generally rectangular advertisements, often approximately 1 to 2 inches high and 3 to 5 inches wide or larger, placed in high-traffic areas of the Internet. Clicking on a banner advertisement results in a transfer to the web page of the advertiser's specification. The sale of banner advertisements is a major source of revenue for online entities, similar to the sale of radio and television advertising time. Banner advertisements are sold by quantities of "impressions," or the number of times an advertisement appears on the web page. Usually, an advertiser purchases thousands or millions of impressions. Popular locations for banner advertising include gay.com, AOL, and similar venues. 
In San Francisco, SFDPH conducted on online banner-advertising campaign on gay.com and on AOL. Nine separate advertisements were run, for a total of over 33 million impressions. The advertisements yielded 32,270 clicks to SFDPH websites with syphilis information, for a click-through rate of $0.1 \%$. The cost per click-through ranged from $\$ 0.05$ to $\$ 10$, depending on the host site and ad placement. Data on the amount of time spent on SFDPH websites are not available for this report.

Some organizations can be persuaded to provide online advertising for reduced or no cost as a service to the public-health community. This practice has not been widespread among online venue owners. However, in recent months, officials from manhunt. net, an online sex-seeking venue, have contacted local public health partners in Houston (i.e., Montrose Clinic) to offer some free services such as online accounts for outreach workers, banner advertising, etc. Manhunt.net staff continue to contact local health departments in areas to which they are expanding their market. The company reports mixed responses from health departments, with some not returning phone calls.

\section{Interactive, Targeted Interventions}

Online interventions can be differentiated from chat-room outreach and from standard, didactic, education websites in that they are more interactive and targeted to the user. Typically, an intervention begins with the participant answering some questions about risk behaviors. The software running the intervention then provides tailored, targeted feedback to the visitor, based upon preprogrammed algorithms created by sexual health experts.

In Houston, staff are developing an online self-interview, analogous to the syphilis case-management interview typically conducted in person by the DIS. This system is being designed for participants who cannot or will not work with DIS to provide risk behaviors in a one-on-one, face-to-face setting. The interview will include partner elicitation, venue elicitation, and questions about risk that are generally included in case-management interviews. The system can be available to patients diagnosed with gonorrhea, chlamydia, and HIV as well. Finally, the interview may prove useful in facilitating partner notification for clients of private providers who typically do not provide this service. These providers can give their patients a card, detailing the interview website and providing instructions. The patients then can initiate the partner services themselves.

One online, targeted, interactive intervention for MSM has been developed and tested in the United States. The intervention, called SmartSexQuest, ${ }^{9}$ aimed to increase STD and HIV testing among MSM and increase condom use with nonmain partners. Though men were willing to $\log$ on and read through the intervention materials, few (15\%) returned to complete the follow-up questionnaire after 3 months. Thus, it is difficult to determine what effect on risk behavior, if any, the intervention had. International efforts to develop and test online interventions, though beyond the scope of this paper, have been conducted. ${ }^{11,12}$

\section{Discussion}

Clearly, the Internet presents significant public-health challenges but could yield vast benefits if harnessed properly. Adapting current, in-person, syphilis-control programs to the online milieu will be a significant task. At present, some obstacles to accomplishing this task include local policies restricting Internet use by state and local officials; a lack of available computer equipment with unrestricted Internet access; training and staffing needs; privacy and security considerations; and difficulty evaluating the success and cost-effectiveness of online programs.
Evaluation of online programs is an urgent need. For example, though many sites have implemented chat-room outreach, it is unclear whether this activity can be recommended for public health departments across the nation. Chat-room outreach requires staff members to spend hours each day in chat rooms, often in "passive mode," hoping to connect with MSM at risk for syphilis. Though some chat-room outreach efforts have been successful at bringing clients to the STD clinics, it is unclear whether the number of cases detected can justify the staff time spent on this activity.

Similarly, banner advertising should be evaluated to determine whether the outcome is worth the cost of advertising. Results will vary, depending on what the banners are designed to advertise. If a banner simply sends visitors to a didactic, educational website, the benefit is difficult to gauge. However, if a banner sends visitors to a site offering coupons for syphilis testing, evaluation may be simpler. In the case of online partner notification, data should be gathered to show that these efforts are successful (i.e., partners can get notified, tested, and treated) without engendering privacy violations. These evaluations should be fairly straightforward, and yet data on online partner notification are not widely available.

Collaborations with online venue owners to promote structural change in the venues may be useful. For example, online sexseeking venues could require users to indicate the last time they were tested for STD and HIV, providing a "don't want to answer" or "ask me in person" option for privacy considerations. While this does not guarantee honesty or disclosure on the part of sex seekers, it may provide an impetus for men to address these issues before having sex. Venue owners also may provide free accounts to public-health officials for the purposes of chat-room outreach and partner notification. Finally, if DIS are aware of an outbreak occurring in online venues, the venue owners may be willing to take responsibility for publishing health-related notices to sex seekers. However, a current problem with addressing venue owners is that many health officials are contacting the owners of several different venues with a variety of requests. This creates a burden on both sides of the issue. One solution may be the use of a national group representing public health officials who can negotiate with online venue owners in a more direct manner.

Finally, the field clearly needs guidance on, and assistance with, bringing local public health programs into the Internet age. This should include equipment, training, technical assistance, changes in local policy to allow access to the Internet, changes in data management strategies to accommodate online records, development of protocols for the protection of privacy and security, development of evaluation plans, and changes in staff configuration. Though these are comprehensive and far-reaching changes, they are necessary for addressing the pervasive public health challenges brought about by the Internet.

\section{References}

1. Bull SS, McFarlane M. Soliciting sex on the Internet: what are the risks for sexually transmitted diseases and HIV? Sex Transm Dis 2000; 27:545-550.

2. McFarlane M, Bull SS, Rietmeijer CA. The Internet as a newly emerging risk environment for sexually transmitted diseases. JAMA 2000; 284:443-446.

3. CDC. Internet use and early syphilis infection among men who have sex with men: San Francisco, California, 1999-2003. MMWR Morb Mortal Wkly Rep 2003; 52:1229-1232.

4. Ashton M, Sopwith W, Clark P, McKevley D, Lighton L, Mandal D. An outbreak no longer: factors contributing to the return of syphilis in Greater Manchester. Sex Transm Infect 2003; 79:291-293.

5. Klausner JD, Wolf W, Fischer-Ponce L, Zolt I, Katz MH. Tracing a syphilis outbreak through cyberspace. JAMA 2000; 284:447-449. 
6. CDC Internal reports (2000-2003). PDSB Thursday reports

7. Klausner JD, Levine DK, Kent CK. Internet-based site-specific interventions for syphilis prevention among gay and bisexual men. AIDS Care 2004; 16:964-970.

8. Constant P. Internet use and partner notification in a cluster of HIV and other STD among men who have sex with men. Abstract presented at the STD/HIV Prevention and the Internet Conference, Washington, DC, 2003

9. Bull SS, Lloyd L, Rietmeijer CA, McFarlane M. Recruitment and retention of an online sample for an STD/HIV prevention intervention targeting men who have sex with men. AIDS Care 2004; 16:931-943.
10. CDC. Using the Internet for partner notification of sexually transmitted diseases: Los Angeles County, 2003. MMWR Morb Mortal Wkly Rep 2004; 53:129-131.

11. Davidovich U, deWit J, Stroebe W. The effect of an internet intervention for promoting safe sex between steady male partners: results of a longitudinal randomized controlled trial online. Paper presented at the XV International AIDS Conference, Bangkok, Thailand, 2004.

12. Davidovich U, deWit J, Stroebe W. (2004). High dropout rates during online randomized controlled trials evaluating HIV-prevention interventions on the Internet -lessons for future online longitudinal studies. Paper presented at the XV International AIDS Conference, Bangkok, Thailand. 